\title{
Aetiology of late-life depression
}

\author{
Robert Baldwin
}

Depressive disorder has no single cause. Arguably, in old age it is a more heterogeneous condition than in earlier adult life, in both clinical presentation and causation. A broad distinction is often made between early- and late-onset depression in later life. The aetiology of these may differ and is currently the subject of new research.

Aetiology is often subdivided into risk factors (vulnerability to depression), precipitating factors and perpetuating factors (features which maintain depression). Perpetuating factors overlap with management issues and will not be discussed in detail. Lastly, there are factors which protect (or 'buffer') against depression.

\section{Risk factors}

\section{Genetic susceptibility}

In general, the later the onset of a depressive disorder, the less significant the role of genetic factors in its aetiology. Hopkinson (1964) found that the prevalence of depressive disorder among first-degree relatives of individuals with depression whose illness began after the age of 50 was less than half of that of probands with early-onset depression.

\section{Gender and civil status}

In cross-sectional studies women, widows and widowers and divorcees have been shown to be at greatest risk (Kivela et al, 1988).

\section{Neurotransmitter changes}

Normal ageing is associated with decreased brain concentrations of serotonin, dopamine, noradrenaline and their metabolites (Veith \& Raskind, 1988), which would be expected to predispose to depression. However, turnover and availability also change with age, so that the picture is not straightforward. A decrease in plasma 3-methoxy-4-hydroxyphenylglycol (MHPG) (suggestive of increased noradrenaline turnover) may be associated with late-life depression (Karege et al, 1989) and the 5-hydroxytryptamine (5HT) metabolite 5-hydroxyindoleacetic acid (5HIAA) has consistently been shown to increase with age (Karlsson, 1993) suggesting hyperactivity in serotonergic pathways. However, decreased levels of 5-HIAA in cerebrospinal fluid (CSF) may correlate more closely with certain symptoms of depression notably anxiety and suicidal behaviour - than age of onset. Furthermore, $5-\mathrm{HT}_{1}$ and 5- $\mathrm{HT}_{2}$ binding sites decrease in number with age, whereas they appear to increase in depressive disorders (Sparks, 1989). Changes with ageing in dopaminergic function such as reductions of CSF homovanillic acid (HVA) levels and increased brain, plasma and platelet monoamine oxidase $B$ (MAO-B) activity have all been shown and these could predispose to depression (Veith \& Raskind, 1988) - although other evidence suggests that platelet MAO activity may correlate with severity and certain symptoms of depression (e.g. anhedonia) more than age of depression onset (Schneider, 1992). In one study of elderly females with depression, those with depression onset before 55 years had lower platelet MAO activity than those with later onset (Alexopoulos et al, 1984). Also, there

Robert Baldwin became Consultant in Old Age Psychiatry at Manchester Royal Infirmary in 1985 and was appointed Honorary Senior Lecturer in 1996 (York House, Manchester Royal Infirmary, Oxford Road, Mancester M13 9BX. Tel. 0161 276 5317; fax 0161276 5303; e-mail Rbaldwin@fs1.cmht.nwest.nhs.uk). His interests and published work include prognosis of depressionin later life, neuroimaging abnormalities in depression, detection of mood disorders in the elderly in liaison settings, and services for young people with dementia. 
is evidence of decrease in platelet 5-HT uptake sites in elderly patients with depression (Schneider 1992). Lastly, neuropeptides may act as neurotransmitter modulators but there is no convincing evidence that their levels alter significantly with age (Leake \& Ferrier, 1993). Table 1 summarises the data. It is clear that some but by no means all age-associated changes are similar to those which occur in depression.

\section{Neuroendocrine changes}

At all ages, depression is associated with hyperactivity and dysregulation of the hypothalamicpituitary-adrenal (HPA) axis. Ageing is associated with increasing cortisol levels and cortisol nonsuppression. Schneider (1992) has speculated that normal ageing is associated with enhanced limbicHPA axis activity, perhaps related to neuronal degeneration in the hippocampus, and that the latter may be exacerbated by raised glucocorticoid secretion, caused either by depression or repeated stressful life events or both (the 'feed-forward cascade'). In support, Krishnan (1991) reported reduced $T_{1}$ spin-lattice relaxation times in the hippocampus, as measured by magnetic resonance imaging (MRI), in a group of patients with depression compared with controls. The effect was particularly striking for elderly patients and was suggestive of tissue damage. The TSH (thyroidstimulating hormone, thyrotropin) response to administered thyroid-releasing hormone is less agedependent but is not specific for depression and is quite variable in ageing persons (Schneider, 1992).

Table 1. Neurotransmitter changes in depression and ageing: direction of change in key markers

$\begin{array}{lcc}\text { Markers } & \text { Ageing } & \text { Depression } \\ \text { MHPG (NA) } & \uparrow & \downarrow \\ \beta \text {-receptors } & \downarrow & \uparrow \\ \alpha_{2} \text {-receptors } & \downarrow \text { or normal } & \uparrow \\ \text { 5-HIAA (5-HT) } & \uparrow & \downarrow \\ \text { 5-HT } 2 \text { receptors } & \downarrow & \uparrow \\ \text { HVA (DA) } & \downarrow & \downarrow \\ \text { MAO-B } & \uparrow & \uparrow \\ \text { Acetylcholine } & \downarrow & ? \\ \begin{array}{l}\uparrow, \text { increased; } \downarrow \text {, decreased; NA, noradrenaline; DA, } \\ \text { dopamine; MHPG, 3-methoxy-4-hydroxyphenylglycol; }\end{array} \\ \begin{array}{l}\text { 5-HIAA, 5-hydroxyindoleacetic acid; HVA, homovanillic } \\ \text { acid; MAO-B, monoamine oxidase B }\end{array}\end{array}$

The dexamethasone suppression test (Carroll et $a l, 1981)$ has not proved a reliable aetiological marker of late-onset depression. It cannot reliably differentiate depression from dementia and is influenced by a host of medical conditions and drugs.

Recent studies of the corticotropin-releasing hormone (CRH) have shown that CRH mRNA levels in the paraventricular nucleus of elderly patients with depression are higher than the levels in patients with Alzheimer's disease (also elevated), and very much higher than in normal controls (Raadsheer $e t$ $a l, 1995)$. This has led to speculation that hyperactivation of paraventricular $\mathrm{CRH}$ neurons may contribute to the aetiology of late-life depression.

\section{Structural change}

\section{Volumetric change}

Generalised atrophic changes in late-life depression are suggested by prominence of cerebral sulci and increased ventricular size. Some studies have reported focal volume reductions in the frontal lobes (Coffey et al, 1993) and the caudate nucleus (Krishnan et al, 1992).

It is unclear whether such changes predispose to depression or whether depression causes atrophy. Possible mechanisms include hypercorticolism, malnutrition or weight loss. Electroconvulsive therapy (ECT) is also associated with cerebral atrophy (Coffey et al, 1991) but it is not thought that ECT itself is responsible for this (Devanand et al, 1994).

\section{White matter and other lesions}

High-intensity signals (hyperintensities) in the white matter, visualised using MRI, are very common in late-life depression; so too are subcortical changes involving the basal ganglia and caudate (summarised in Baldwin, 1993) (see Fig. 1). Silent stroke is also common (Fujikawa et al, 1993).

\section{The vascular depression hypothesis}

This is based on a number of premises (discussed in more detail by Alexopoulos et al, 1997) (see Box 1). First, patients with vascular disease have a high rate of depression. Second, diseases affecting blood vessels, such as stroke, hypertension and diabetes, are associated with frequent white matter hyperintensities (WMH). Third, neurological diseases affecting the subcortex are associated with a high rate of depression. Fourth, late-onset depression may be associated with higher rates of vascular disease and vascular risk factors compared with early-onset 


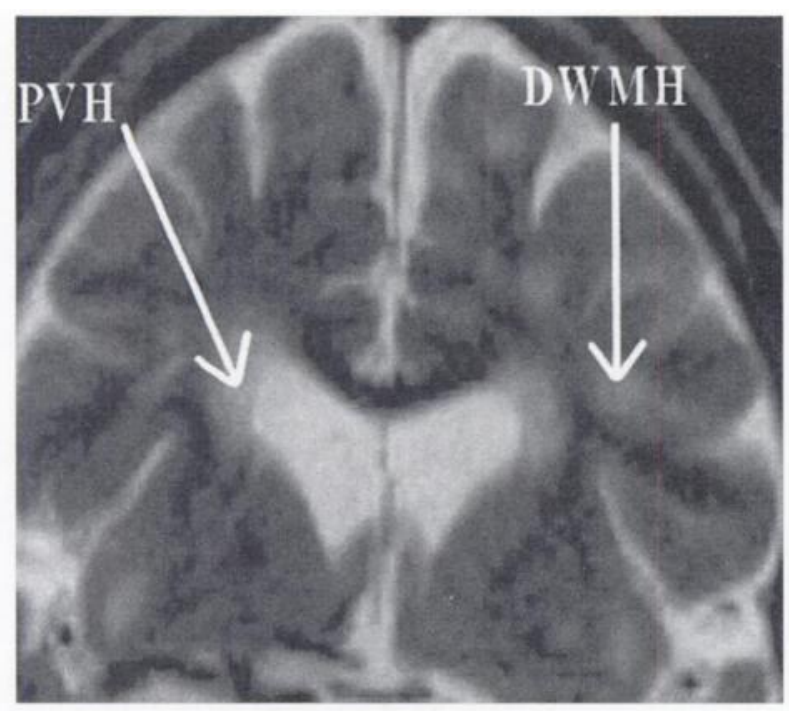

Fig. 1. $\mathrm{A} \mathrm{T}_{2}$-weighted magnetic resonance imaging scan illustrates some of the changes which may be important in the aetiology of late-life depression. Abnormal signals appear white (high signal intensity). In a coronal section (above), periventricular hyperintensities (PVH) around the lateral ventricles and in the deep white matter (DWMH) can be seen. In a transverse section (right), subcortical grey matter lesions are apparent and involve the caudate, pallidum and thalamus (from top to bottom). These changes may disrupt the striato-pallidal pathway which may be critical in mood regulation (courtesy of Steven Simpson, The Fortson Clinic, Dorset)

Box 1. Vascular depression hypothesis

\section{Premises}

Vascular disorders are associated with depression Hyperintensities on MRI associated with vascular disorder

Subcortical disease is associated with depression Late-onset depression associated with greater vascular disease and vascular risk

Late-onset depression associated with more deep white matter abnormality than early-onset depression in later life

Proposed features

Mainly late-onset

Reduced depressive ideation

Reduced insight

More overall morbidity

Apathy and retardation

More cognitive impairment (particularly executive dysfunction)

Poorer recovery

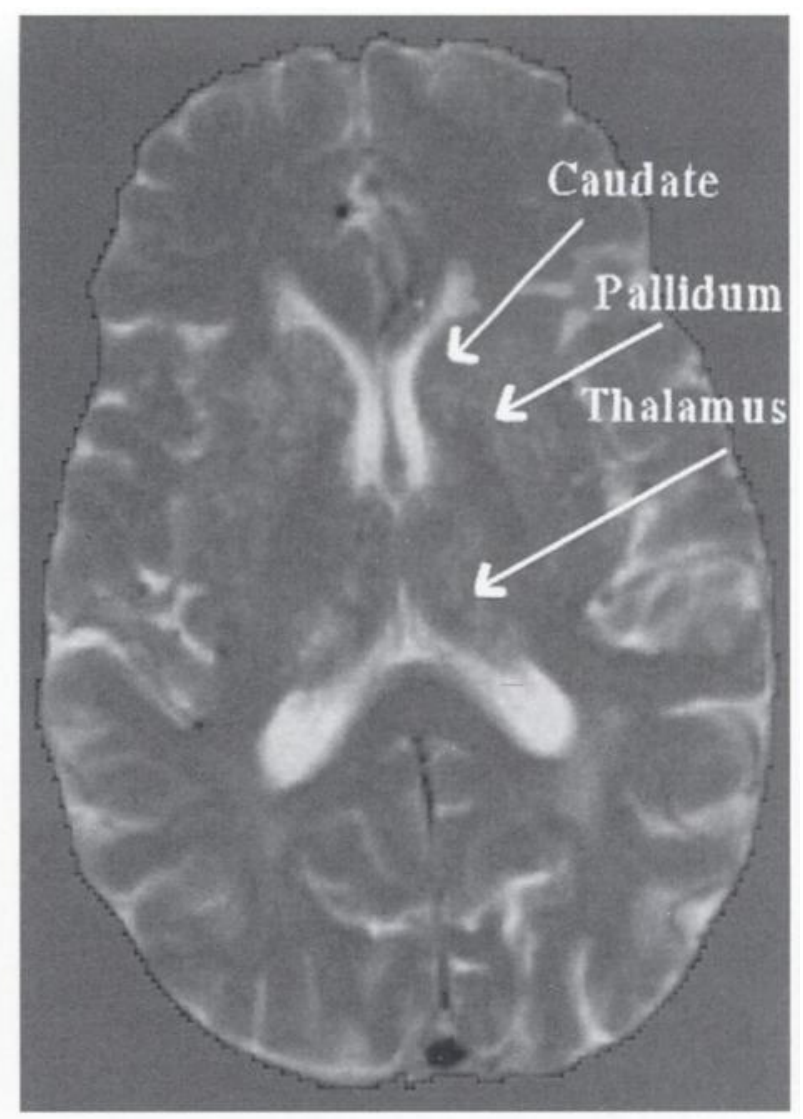

depression in late life and with higher rates of $\mathrm{WMH}$ (Baldwin \& Tomenson, 1995).

In 'vascular depression', damage to end-arteries supplying subcortical striato-pallido-thalamocortical pathways disrupts neurotransmitter circuitry involved in mood regulation and may thus cause or predispose to depression.

However, the significance of WMH is controversial. Although more common in elderly people with depression, $\mathrm{WMH}$ is not confined to older people or to depression itself (Baldwin, 1993) and may occur in a wide variety of non-vascular disorders such as hydrocephalus, multiple sclerosis, Alzheimer's disease and in normal ageing. A study of late-life depression (Lyness et al, 1998) failed to demonstrate any relationship between cerebrovascular disease risk factors and depression severity or symptomatology. In another study (Kumar et al, 1997a), brain volume measures were associated with general measures of physical ill health rather than specifically cardiovascular disease or cardiovascular risk factors. So, it cannot be assumed uncritically that visualised brain changes are caused by end-artery disease.

Whether 'vascular' or not in aetiology, there is evidence implicating WMH and subcortical pathology in late-life depression with: subtypes of late-life depression such as psychotic depression ( $\mathrm{O}^{\prime}$ Brien et al, 1997) and 'minor' depression (Kumar et al, 1997b); the presence of particular symptoms 
(reduced depressive ideation and increased psychomotor retardation (Alexopoulos et al, 1997); more overall physical morbidity (Kumar et al, 1997a); and a poorer outcome with antidepressant treatment (Simpson et al, 1998).

The vascular hypothesis, although unproven, is an important aetiological concept as it lends itself to hypothesis-driven research which can be supported by modern neuroimaging techniques. It also has important implications for the treatment of depression. These include whether agents to reduce the risk of end-artery occlusion, such as aspirin, may be of use, as well as rehabilitative strategies designed to counter brain-based apathy and lack of motivation.

\section{Dynamic changes}

There is a general reduction in cerebral perfusion mainly to the frontal cortex (Sackheim et al, 1990). However, it is not known to what extent these changes are state or trait.

\section{Depression and dementia}

Neuronal loss is greater in the locus coeruleus of patients with dementia who are depressed compared with those without depression (Forstl et al, 1992). This suggests a distinct pathological substrate for depression in these patients. Depressed patients without dementia but with cognitive impairment have reduced flow to the left anterior medial prefrontal gryus and increased flow to the cerebellar vermis (Bench et al, 1992), supporting a neurobiological basis for 'depressive pseudodementia', which most often arises in the context of later-life depression.

\section{Personality}

Research has emphasised the presence of obsessional traits, 'avoidant' and 'dependent' types of personality and a difficulty in forming close confiding relationships as possible aetiological factors in late-life depression (Murphy, 1982).

Bergmann (1978) has adapted attachment theory to the aetiology of late-life depression. He suggests that satisfactory attachment behaviour in early life is a necessary prerequisite to later adaptive coping, rather than non-adaptive anxiety, in the face of the very real threats and losses which accompany old age.

\section{Physical health and disability}

Both hospital (Murphy, 1982) and community (Prince et al, 1998) series reveal a close association between the onset of depression and physical ill health. Physical impairment may provoke a depressive disorder, which may in turn increase the degree of disability associated with the original physical impairment (Prince et al, 1998).

There are several mechanisms whereby physical disorder may lead to depression. The dementias, stroke and Parkinson's disease are all associated with depression. Specific brain-based aetiologies have been proposed but discussion is beyond the scope of this review. These more biologically-oriented explanations may help us to understand how a depressive episode (depressive illness) could arise in the setting of particular disorders. They are less helpful in clarifying the onset of depressive symptoms, which occur commonly in many illnesses, including many non-neurological ones. The wide range of medical conditions, as well as hearing and visual deficits, which are associated with depression, suggests that the meaning of the illness for the sufferer is as important as the precise body system involved (Murphy, 1982). Prince et al (1998) have shown that disablement, and more specifically handicap - the disadvantage imposed by a physical impairment and disability - is the most important risk factor for depression in older people at home.

The highest prevalence of depressive disorders in older people is found in residential and nursing homes, medical wards for the elderly and among older people in receipt of extensive home care support (Ames, 1988; Banerjee et al, 1996). The usual explanation is that this simply reflects the concentration of disablement found in these settings. However, the concept of handicap (the disadvantage to the individual caused by a disability) provides another perspective. It is possible that some caring environments disadvantage older people by depriving them of benefits available to others with a similar level of disability who reside elsewhere. Some care settings may therefore be aetiological agents for depression. This has implications for prevention.

Lastly, a large number of physical disorders (some occult at presentation of depression), drugs and iatrogenic factors are associated with the onset of depression. The main ones are listed in Table 2 . The elderly are particularly prone.

\section{Care-giving}

This deserves special attention as a risk factor of depression, as there are opportunities for prevention, identification and treatment. In one study (Ballard et al, 1996) a quarter of carers of people with dementia were depressed, and many had persistent symptoms. Factors associated with depression in carers were 
depression in the designated patient and problem behaviours arising from him or her.

\section{Epidemiological findings}

Epidemiological research has not demonstrated that ageing per se is associated with an increased risk of developing depression (Robert et al, 1997). Any apparent age effect is explained by the additional risk caused by disability and ill health in those with depression (Robert et al, 1997; Prince et al, 1998).

Palsson et al (1999) found that measures of brain atrophy were similar in depressed and mentally healthy older subjects derived from a population sample, so it may be unwise to extrapolate from hospital studies, which comprise most of those cited in this review, to the community at large. However, in an epidemiological study, Cervilla \& Prince (1997) found associations between depression and exposure to social supports deficits and threatening life events, which were considerably stronger for subjects without cognitive impairment than for those with it. This lends support to two pathways to depression in the elderly, one via organic brain disease and another via social stress and deficient social support.

Incident studies are useful in separating factors which might be aetiological from those which are of prognostic importance. An example is gender.
Although most cross-sectional studies find that female gender is a risk factor, in one study (Schoevers et al, 1999) this was not true. An explanation is that gender is a prognostic and not a risk factor. Female gender may be associated with chronicity, which may inflate the finding of a gender bias in crosssectional research.

Green et al (1992) found that lack of life satisfaction, feelings of loneliness, and smoking were associated with depression. Multivariate analysis identified loss of someone close within the previous six months as a further factor. In a prospective community study, Schoevers et al (1999) identified bereavement of a spouse, health problems and comorbid organic deterioration (from baseline) as risk factors for the incidence of depression. More complex interactions were noted. For example, being married or having adequate social support significantly reduced the impact of reduced functional ability (at baseline) in triggering depression over time.

\section{Precipitating factors}

Box 2 lists the main types of precipitants, which can be categorised as either recent life events or chronic stressful conditions. Murphy (1982) found that $48 \%$

Table 2. Medical illnesses and centrally acting drugs that may cause organic mood syndromes
Medical conditions
Endocrine/metabolic
Hypo-/hyperthyroidism
Cushing's disease
Hypercalcaemia
Sub-nutrition
Pernicious anaemia
Organic brain disease
Cerebrovascular disease/stroke
Central nervous system tumours
Parkinson's disease
Alzheimer's disease
Multiple sclerosis
Systemic lupus erythematosus
Occult carcinoma
Pancreas
Lung
Chronic infections
Neurosyphilis
Brucellosis
Neurocysticercosis
Myalgic encephalomyelitis
Acquired immune deficiency syndrome

\section{Centrally acting drugs}

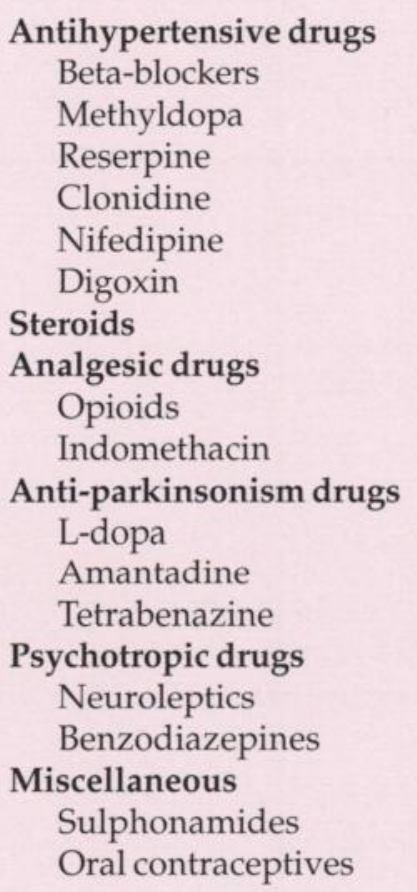

Antihypertensive drugs

Beta-blockers

Reserpine

Clonidine

Nifedipine

Digoxin

Steroids

nalgesic dru

Opioids

Indomethacin

L-dopa

Amantadine

Neuroleptics

Benzodiazepines

Sulphonamides

Oral contraceptives 
Box 2. Precipitating factors in depression of later life

\author{
Life events \\ Bereavement \\ Separation \\ Acute physical illness \\ Medical illness or threat to life of someone close \\ Sudden homelessness or having to move \\ into an institution \\ Major financial crisis \\ Negative interactions with family member \\ or friend \\ Loss of 'significant other' (including a pet)
}

Chronic stress

Declining health and mobility; dependence

Sensory loss; cognitive decline

Housing problems

Major problems affecting family member

Marital difficulties

Socio-economic decline

Problems at work; retirement

Caring for a chronically ill and dependent family member of elderly patients with depressive disorder $(n=119)$ had experienced at least one severe life event in the preceding year, compared with $23 \%$ of a control group. Neither these percentages nor the types of adverse events are very different from other adult age groups, save for ill health, which is a more common precipitant in older people. Major social difficulties (as distinct from abrupt life events) lasting for two years or more were also significantly associated with depression.

\section{Protective factors}

Several factors - biological, psychological and social - seem to exert a buffering effect (see Box 3 ). They may therefore protect an older person from developing depression in the face of the precipitating events discussed above.

Box 3. Protective (buffering) factors

\section{General medical care}

Correcting physical deficits (eg sensory loss)

Optimising general health

Good nutrition

Physical fitness

\section{Coping behaviours}

Adaptive, integrated personality

Capacity for confiding relationships

Active coping styles to overcome adversity (as opposed to helplessness)

\section{Social supports}

Adequate social network

Tangible social support

Positive perceptions of support

Confiding relationships

Religious/spiritual beliefs
Since ill health is a major risk factor for depressive disorder in older people, maintaining optimum health is a worthwhile goal. Although evidence is scant, there is an emerging view that fitness and exercise programmes may play a preventive role in older people. Individuals with more integrated personalities and active coping styles are less prone to depression, as are those with robust social support systems - whether informal (Murphy, 1982), familyoriented or formal (Koenig et al, 1998) - although it is probably the perception (that is, quality) of support that matters more than the actual amount of it.

\section{Factors in relapse and recurrence}

Depression is prone to relapse and recurrence. Hence, a previous history of depression is a potent risk factor (Schoevers et al, 1999). Factors implicated in relapse or recovery are best considered as prognostic rather than aetiological; among the more important are treatment adequacy in the continuation and maintenance phases, respectively.

\section{Conclusions}

Each episode of depression arises out of a unique combination of a personal susceptibility to depression and, usually, a precipitant or several precipitants which trigger it. "My boss always taught me to ask, 'Why has this person developed depression now?" ". This apocryphal remark makes good clinical sense -it prompts enquiry into not only the biological realm but also the psychosocial. Another aphorism is "Depression is depression at any age". Largely, this is true, but there is emerging evidence 
of a subtype of late-life depression, often with vascular rather than genetic risk, associated with late age of onset, deep white matter hyperintensities on MRI and subcortical changes. The clinical picture is one of apathy, limited depressive ideation, poor insight and high morbidity. The vascular hypothesis is not proven but generates testable hypotheses of aetiology, which, if supported, would lead to innovative treatments in late-life depression. Although there is some overlap in changes in neurotransmitters with ageing and in later-life depression, there are also inconsistencies, and epidemiological data do not, in any case, support ageing as a risk factor for depression. The search for a specific marker, such as a neuroendocrine test, has so far been disappointing. Rather, those most at risk of depression are socially isolated elderly people, those with sensory impairment and others with significant handicap from physical disorder. Currently, there is probably an overemphasis on the biological causation of late-life depression, and there are dangers in drawing too many conclusions from unrepresentative samples. Epidemiological data may provide a corrective. Emerging data suggest different pathways to depression, in which the impact of stressors known to trigger depression may be modified by patterns of vulnerability which may vary from individual to individual. Proposed factors include genetic, organic and environmental ones.

\section{References}

Alexopoulos, G. S., Lieberman, K. W. \& Young, R. C. (1984) Platelet MAO activity and age at onset of depression in elderly depressed women American Journal of Psychiatry, 141, 1276-1278.

-, Meyers, B. S., Young, R. C., et al (1997) 'Vascular depression' hypothesis. Archives of General Psychiatry, 54, 915-922.

Ames, D., Ashby, D., Mann, A. H., et al (1988) Psychiatric illness in elderly residents of part III homes in one London borough: prognosis and review. Age and Ageing, 17, 249256.

Baldwin, R. C. (1993). Late life depression and structural brain changes: A review of recent magnetic resonance imaging research. International Journal of Geriatric Psychiatry, 8, 115-123.

- \& Tomenson, B. (1995) Depression in later life. A comparison of symptoms and risk factors in early and late onset cases. British Journal of Psychiatry, 167, 649-652.

Ballard, C. G., Eastwood, C., Gahir, M., et al (1996) A followup study of depression in the carers of dementia sufferers. British Medical Journal, 312, 947.

Banerjee, S., Shamash, K., Macdonald, A. J. D., et al (1996) Randomised controlled trial of intervention by psychogeriatric team on depression in frail elderly people. British Medical Journal, 313, 1058-1061.

Bench, C. J, Friston, K. J., Brown, R. G., et al (1992) The anatomy of depression - focal abnormalities of cerebral blood flow in major depression. Psychological Medicine, 22, 607-615.

Bergmann, K. (1978) Neurosis and personality disorder in old age. In Studies in Geriatric Psychiatry (eds A. D. Isaacs \& F. Post), Pp. 41-75. New York: John Wiley \& Sons.

Carroll, B. J. Feinberg, M. Greden, J. F., et al (1981) A specific laboratory test for the diagnosis of melancholia. Archives of General Psychiatry, 38, 15-22.
Cervilla, J. A. \& Prince, M. J. (1997) Cognitive impairment and social distress as different pathways to depression in the elderly: a cross-sectional study. International Journal of Geriatric Psychiatry, 12, 995-1000.

Coffey, C. E., Weiner, R. D., Djang, W. T., et al (1991) Brain anatomic effects of electroconvulsive therapy. Archives of General Psychiatry, 48, 1013-1021.

-, Wilkinson, W. E., Weiner, R. D., et al (1993) Quantitataive cerebral anatomy in depression: a controlled magnetic resonance study. Archives of General Psychiatry, 50, 7-15.

Devanand, D. P., Andrew, J. W., Hutchinson, E. R., et al (1994) Does ECT alter brain structure? American Journal of Psychiatry, 151, 957-970.

Forstl, H., Burns, A., Luthbert, P., et al (1992) Clinical and neuropathological correlates of depression in Alzheimer's disease. Psychological Medicine, 22, 877-884.

Fujikawa, T., Yamawaki, S. \& Touhouda, Y. (1993) Incidence of silent cerebral infarction in patients with major depression. Stroke, 24, 1631-1634.

Green, B. H., Copeland, J. R. M. \& Dewey, M. E. (1992) Risk factors for depression in elderly people: a prospective study. Acta Psychiatrica Scandinavica, 86, 213-217.

Hopkinson, G. (1964) A genetic study of affective illness in patients over 50. British Journal of Psychiatry, 110, 244-254.

Karege, F. Bovier, P. \& Gaillard, J. M. (1989) Plasma MHPG and age of onset in depressed patients. Psychiatry Research, 30, 103-105.

Karlsson, I. (1993) Neurotransmitter changes in aging and dementia. Nordic Journal of Psychiatry, 47 (suppl.), 41-44.

Kivela, S.-L., Pahkala, K. \& Laippala, P. (1988) Prevalence of depression in an elderly Finnish population. Acta Psychiatrica Scandinavica, 78, 401-413.

Koenig, H. G., George, L. K. \& Peerson, B. L. (1998) Religiosity and remission of depression in medically ill older patients. American Journal of Psychiatry, 155, 536-542.

Krishnan K. K. R. (1991) Organic bases of depression in the elderly. Annual Reviews of Medicine, 42, 261-266.

-, McDonald, W. M., Escalona, P. R., et al (1992) Magnetic resonance imaging of the caudate nuclei in depression. Archives of General Psychiatry, 49, 553-557.

Kumar, A., Miller, D., Ewbank, D., Yousen, D., et al (1997a) Quantitative anatomical measures and comorbid medical illness in late-life depression. American Journal of geriatric Psychiatry 5, 15-25.

-, Schweizer, E., Zhisong, M. S., et al (1997b) Neuroanatomic substrates of minor depression. Archives of Neurology, 54, 613-617.

Leake, A. \& Ferrier, I. C. (1993) Alterations in neuropeptides in aging and disease. Drugs and Aging, 3, 408-427.

Lyness, J. M., Caine, E. D., Cox, C., et al (1998) Cerebrovascular risk factors and late-life major depression. American Journal of Geriatric Psychiatry, 6, 5-13.

Murphy, E. (1982) Social origins of depression in old age British Journal of Psychiatry, 141, 135-142.

O'Brien, J. T., Ames, D., Schwietzer, I., et al (1997) Clinical, magnetic resonance imaging and endocrine differences between delusional non-delusional depression in the elderly. International Journal of Geriatric Psychiatry, 12, 211-218.

Pálsson, S., Aevarsson, O. \& Skoog, I. (1999) Depression, cerebral atrophy, cognitive performance and incidence of dementia. Population study of 85-year-olds. British Journal of Psychiatry, 174, 249-253.

Prince, M. J., Harwood, R. H., Thomas, A, et al (1998) A prospective population-based cohort study of the effects of disablement and social milieu on the onset and maintenance of late-life depression. The Gospel Oak Project VII. Psychological Medicine, 28, 337-350.

Raadsheer, F. C., Joop, J., Van Heerikhuize, J. J., et al (1995) Corticotropin-releasing hormone mRNA levels in the paraventricular nucleus of patients with Alzheimer's disease and depression. Archives of General Psychiatry, 152, 1372-1376.

Robert, R. E., Kaplan, G. A., Shema, S. J., et al (1997) Does growing old increase the risk for depression? Americam Journal of Psychiatry, 154, 1384-1390.

Sackeim, H. A., Prohovnik, I., Moeller, J. R., et al (1990) Regional cerebral blood flow in mood disorders 1 . Comparison of major depressives and normal controls at rest. Archives of General Psychiatry, 47, 60-70. 
Schneider, L. S. (1992) Psychobiologic features of geriatric affective disorders. Clinics in Geriatric Medicine, 8, 253265.

Schoevers, R. A., Beekman, A. T. F., Deeg, D. J. H., et al (1999) Risk factors for depression in later life: results of a prospective community based study (AMSTEL). Journal of Affective Disorders, in press.

Simpson, S., Baldwin, R. C., Jackson, A., et al (1998) Is subcortical disease associated with a poor response to antidepressant treatment? Neurological, neuropsychological and neuroimaging findings. Psychological Medicine, $28,1015-1026$.

Sparks, L. D. (1989) Aging and Alzheimer's disease. Altered cortical serotonergic binding. Archives of Neurology, 46, 138140.

Veith, R. C. \& Raskind, M. A. (1988) The neurobiology of aging: does it predispose to depression. Neurobiology of Aging, 9, 101-117.

\section{Multiple choice questions}

1. Risk factors for depression in late-life include:

a a greater genetic risk

b decreased plasma MHPG (3-methoxy-4hydroxyphenylglycol)

c increased platelet monoamine oxidase B activity

d increased platelet serotonin uptake sites

e hypoactivity of the hypothalamic-pituitaryadrenal axis.

2. Structural changes which have been reported in late-life depression include:

a frontal lobe volume reduction

b white matter hyperintensities

c parietal lobe infarction

d reduced volume of the red nucleus

e reduced volume of the caudate nucleus.

3. Symptoms of the newly proposed 'vascular depression' include:
a psychomotor agitation
b increased levels of guilt
c reduced insight
d visual field deficits
e apathy.

4. The following have been shown to be associated with depression onset:
a warfarin
b stroke
c haemophilus pneumonia
d calcium channel blockers
e Alzheimer's disease.

5. The following events have a definite association with onset of later-life depression:
a social difficulties lasting more than two years
b disputes with neighbours
$c$ being a carer
d loss of a pet
e paranoid personality.

MCQ answers

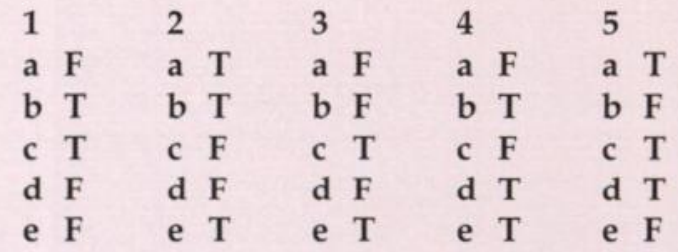

\section{Visit the Royal College of Psychiatrists web site at http://www.rcpsych.ac.uk}

\section{Information on:}

- intitiatives in press and publications conferences library services examinations postgraduate education the College Research Unit

\section{And for members, information on:}

- divisions, faculties, sections, SIGS, committees and more

If you are a member, please e-mail Lucy Alexander to receive your member's password:

lalexander@rcpsych.ac.uk 